\title{
LA SOBREEXPLOTACIÓN DE ACUÍFEROS Y LOS PLANES DE ORDENACIÓN HIDRÁULICA EN LA CUENCA DEL RÍO VINALOPÓ. ALICANTE
}

\author{
Concepción Bru Ronda
}

\section{RESUMEN}

La cuenca del río Vinalopó,con un desarrollo de $1.705 \mathrm{~km}^{2}$, abarca gran porción del límite suroccidental de la provincia de Alicante. Se incluyen en ella 24 municipios, con un total de 384.406 habitantes desigualmente distribuidos.

La penuria hídrica, los altos índices de aridez y la extrema irregularidad de los módulos fluviales determinan unas condiciones limitativas para el mantenimiento de una gran riqueza creada. Aspectos mayorados en los últimos años con la aparición de fenómenos de sobreexplotación de los acuíferos profundos de la cuenca, que abastecían a una gran parte del territorio.

La Administración Hidráulica inició en esta situación las declaraciones provisionales de sobreexplotación de los acuíferos de la Sierra de Crevillente y de Jumilla-Villena. Estas acciones se inscriben en una fase transitoria y preliminar a la aprobación del Plan Hidrológico.

Limitaciones de tipo jurídico y consuetudinario aún no resueltas, así como la propia gravedad de la «situación terminal» de estos acuíferos, que implica otras acciones correctoras no estrictamente ligadas a la ordenación de las extracciones, definen la complejidad en la que se mueven las soluciones al problema hidráulico de la cuenca y al futuro desarrollo que de él depende.

\section{ABSTRACT}

The Vinalopó basin, opened out $1.105 \mathrm{~km}^{2}$, extends to a great share in the southwestern boundary of the province of Alicante. Twenty-four towns are included in it occupying over 384.406 inhabitants unequally distributed.

The hydric shortage, the high rates of aridity and the utmost irregularity of the fluvial modules determine the restrainted conditions for an important created richness. Those circumstances have been emphasized during the last years on the spectre of phenomena of deep aquifer overexploitation that supply a great part of the territory.

The hydraulic Administration, owing to this situation, has started a provisional statement of overexploitation of the Crevillente and Jumilla-Villena aquifers. These prelimi- 
nary actions in a transitory stage precede the approval of the Hydrological Plan of Basin and the National Hydrological Plan.

Legal and customary restrictions, still unresolved, as soon as the seriousness of the «terminal state» of these aquifers, which imply other corrective activities not strictly tied to the planning of water extraction, define the complexity of possible solutions that will result in the future growth and in the hydraulic issue of the Vinalopo basin.

\section{Las condiciones hídricas en la cuenca del Vinalopó}

Con 89 kilómetros de recorrido desde su nacimiento, en el límite NW de la provincia de Alicante, hasta su desagüe difuso en pleno Campo de Elche, ya cerca del Mediterráneo, avena este río una cuenca vertiente de $1.705 \mathrm{~km}^{2}$ de superficie; rasgos que le confieren en el ámbito provincial alicantino el rango de arteria fluvial más importante espacialmente. Sin embargo, la trascendencia hídrica de dicho río-rambla no va pareja a tal desarrollo superficial debido a la incidencia de diversos factores. (GRÁFICO 1)

Situada la cuenca a sotavento de los vientos húmedos del NE, sufre los efectos de una clara sombra pluviométrica, que se hace más evidente desde los observatorios del Alto Vinalopó, con registros de 503,9 mm en Benejama, hasta los del valle medio, con $274 \mathrm{~mm}$ en La Romana, y los $278 \mathrm{~mm}$ ya en al Bajo Vinalopó. Estos totales anuales y los datos medios de precipitaciones mensuales enmarcan una realidad hídrica, la fuerte irregularidad interanual, que combina años extremadamente secos, y a veces consecutivos, con otros de excepcionales y torrenciales lluvias.

Las temperaturas medias oscilan en la cuenca entre los 13,1 grados de Benejama y Sax y los 18,1 de Elche, cifras estas que ofrecen de partida una benignidad térmica, merced a la situación de la zona a sotavento de la Meseta y en contacto con el Mediterráneo.

La capacidad evaporativa de las temperaturas marcan unos estrictos topes entre los cuales se mueve la disponibilidad de los recursos, así como las necesidades hídricas de los cultivos.

Según el método ideado por Thornthwaite para analizar el balance hídrico, en todos los observatorios estudiados la necesidad de agua (ETP) es superior a los $710 \mathrm{~mm}$ y en general es evidente la penuria del índice medio de humedad en los observatorios de la cuenca, ya que exceptuando los más favorecidos (Benejama) el resto adolece de recursos hídricos la mayor parte del año. Así, a excepción de un reducido espacio al $\mathrm{N}$ de la cuenca, donde se da un tipo seco-subhúmedo, el resto de observatorios presenta un índice hídrico semiárido en una franja intermedia o de transición (valle medio), y árido en el sector más meridional de la cuenca.

Con excepción del Alto Vinalopó, y aquí con valores muy reducidos, en el resto de la cuenca la lluvia útil es inexistente: dato que se amplia un poco más si seguimos el método de Turc para calcular este parámetro, pero que muestra saldos claramente deficitarios en el Bajo y parte del Medio Vinalopó.

Para una superficie de $1.705 \mathrm{~km}^{2}$ el balance hídrico de la cuenca, según métodos empíricos, se mueve entre una aportación de 48 y $85,5 \mathrm{Hm}^{3}$, de los cuales circulan epígeamente en proporción un $50 \%$, en especial en su parte alta, ya que la cifra se reduce bastante en su tramo medio y bajo debido a los reducidos índices de permeabilidad que presentan. La poca pendiente de la cuenca, así como la menor pluviometría y las continuas derivaciones son, además de otros factores, las que eliminan en el tramo medio y bajo los flujos superficiales de carácter natural.

La aportación media calculada para una serie larga de años es de $12 \mathrm{Hm}^{3}$, lo que indica un módulo específico de 0,2 1/s. La máxima es de 31,8 (1971-72) y el mínimo de 2,7 
GRÁFICO 1. Localización del área de análisis.

(1912-14). Sin embargo el dato abultado de $31,8 \mathrm{Hm}^{3}$ se debe al aumento en los últimos años de las aguas residuales vertidas al cauce del río en su tramo medio procedentes de la zona industrial y de los efluentes urbanos que se han incrementado merced al aumento poblacional de la cuenca. Estas aguas, que presentan elevadas cotas de contaminación, son las que circulan por el cauce en el tramo medio y bajo hasta su desembocadura en la Albufera de Elche-Santa Pola.

En el Vinalopó la mayor parte de los recursos utilizados actualmente se refieren a aguas subterráneas, que al fin y al cabo forman parte de la lluvia útil que se infiltra hacia los acuíferos profundos.

Las aguas subterráneas constituyen una de las principales fuentes de recursos hídricos de la Unidad del Vinalopó y de otras áreas limítrofes a las que abastece; sin embargo, la paleografía y los aspectos climáticos locales son los principales responsables de los límitados recursos de aguas profundas.

Intentar establecer un balance global de los recursos subterráneos en la cuenca del Vinalopó y en el estado actual de los conocimientos resulta algo arriesgado, ya que las delimitaciones de las formaciones acuíferas que afectan a la cuenca, las posibles interco- 
1 Yecla-Villena-Benejama

2 Peñarrubia

3 Jumilla-Villena

4 Carche-Salinas

5 Argueña-Maigmo

6 Sierra de Quibas

7 Sierra del Cid

8 Crevillente

9 Sierra de Mariola

Alto grado de explotación

$\triangle$ Subexplotado

$\mathbb{H}$ Sobreexplotado

GráfICO 2. Principales sistemas acuíferos y captaciones de aguas subterráneas.

FuENTE: Estudio de la infraestructura hidráulica de la cuenca del Vinalopó (D.G.O.H.-Plan Hidrológico Nacional).

nexiones, así como las trasferencias hidráulicas estan todavía en fase de revisión y estudio. Hay que considerar además el hecho de que cierto volumen de recursos computables se inscriben en espacios extracomarcales, extraprovinciales e incluso pertenecientes a otras cuencas vertientes.

Una aproximación al tema puede establecerse si tenemos en cuenta que los recursos procedentes de la alimentación media anual ascienden, para todos los acuíferos y en conjunto, a $58 \mathrm{Hm}^{3} / a n ̃ o$. Mientras que las cifras de extracciones, y ahora sólo valorando las consumidas en la cuenca, se situan entre 85,39 y 123,9 Hm³/año. Estas cifras nos ofrecen una perspectiva de la situación que refleja unos altos grados de explotación en toda la cuenca, que permiten hablar exclusivamente de un acuífero en equilibrio, el de MariolaPinar de Camus. Otras seis formaciones acuíferas se situan en un alto grado de explotación y existen dos acuíferos declarados provisionalmente sobreexplotados, los de Jumilla-Villena y Crevillente (vide gráfico 2). 


\section{CuAdro I}

EVOLUCIÓN DE LA POBLACIÓN DE LAS COMARCAS DEL VINALOPÓ EN ÉPOCA CENSAL

\begin{tabular}{|c|c|c|c|c|c|c|c|c|c|c|c|}
\hline Municipio: & 1860 & 1900 & 1910 & 1920 & 1930 & 1940 & 1950 & 1960 & 1970 & 1980 & 1990 \\
\hline Bañeres & 2564 & 3317 & 3224 & 3691 & 3698 & 3447 & 3729 & 4967 & 5873 & 6704 & 7085 \\
\hline Benejama & 2014 & 2529 & 2622 & 2328 & 2197 & 2073 & 2183 & 2252 & 2078 & 1944 & 1850 \\
\hline Biar & 3172 & 3550 & 3534 & 3274 & 3287 & 2838 & 2733 & 2748 & 2976 & 3203 & 3412 \\
\hline Campo de Mirra & 798 & 945 & 1014 & 817 & 671 & 596 & 630 & 538 & 505 & 413 & 396 \\
\hline Cañada & 686 & 1036 & 994 & 1103 & 974 & 1010 & 1062 & 1074 & 1121 & 1099 & 1058 \\
\hline Sax & 2856 & 4582 & 4403 & 3852 & 4196 & 3795 & 4065 & 4664 & 5655 & 7336 & 8309 \\
\hline Villena & 10214 & 14099 & 15692 & 16544 & 17638 & 19065 & 19994 & 21934 & 25473 & 28735 & 31037 \\
\hline \multicolumn{12}{|l|}{ Total } \\
\hline Alto Vinalopó & 22304 & 36058 & 31483 & 31609 & 32661 & 32824 & 34386 & 38177 & 43681 & 49434 & 53147 \\
\hline Aspe & 7186 & 7927 & 7961 & 7650 & 7351 & 7812 & 8770 & 10279 & 13229 & 15291 & 16008 \\
\hline Elda & 4085 & 6132 & 8028 & 8078 & 13445 & 20050 & 20699 & 28151 & 41511 & 53128 & 57515 \\
\hline \multicolumn{12}{|l|}{ Hondón de } \\
\hline los Frailes & & & & & 793 & 704 & 668 & 555 & 556 & 528 & 554 \\
\hline \multicolumn{12}{|l|}{ Hondón de las } \\
\hline Nieves & 2116 & 3690 & 3538 & 3454 & 2232 & 1824 & 1936 & 1786 & 1653 & 1562 & 1517 \\
\hline La Algueña & & & & & & 1667 & 1888 & 1722 & 1589 & 1609 & 1527 \\
\hline La Romana & & & & & 2264 & 1984 & 2081 & 2082 & 1996 & 2010 & 1971 \\
\hline Monforte & 3678 & 3798 & 3720 & 3429 & 3205 & 3217 & 3133 & 3437 & 4020 & 4656 & 4943 \\
\hline Monovar & 8426 & 10601 & 11243 & 10377 & 9799 & 9933 & 9979 & 10393 & 10348 & 11140 & 12122 \\
\hline Novelda & 7925 & 11388 & 12045 & 11994 & 9508 & 10349 & 10598 & 12911 & 17344 & 20950 & 22288 \\
\hline Petrel & 2911 & 3928 & 3992 & 4120 & 5209 & 5506 & 6145 & 10615 & 15804 & 20612 & 23591 \\
\hline Pinoso & 4718 & 7946 & 8142 & 8245 & 7740 & 5114 & 6015 & 5194 & 5101 & 5228 & 5674 \\
\hline Salinas & 969 & 1440 & 1261 & 1123 & 1239 & 1108 & 1058 & 1112 & 918 & 1007 & 1096 \\
\hline \multicolumn{12}{|l|}{ Total } \\
\hline Medio Vinalopó & 42014 & 56849 & 59930 & 58470 & 62866 & 69286 & 72970 & 88237 & 114069 & 137711 & 148806 \\
\hline Crevillente & 8284 & 10726 & 10452 & 11216 & 11191 & 11403 & 12636 & 14047 & 16091 & 20940 & 22456 \\
\hline Elche & 18734 & 27308 & 30511 & 33167 & 38013 & 46596 & 55877 & 73320 & 122663 & 164311 & 184912 \\
\hline Santa Pola & 2759 & 4100 & 3935 & 4022 & 4200 & 5325 & 5851 & 6443 & 9198 & 12010 & 14725 \\
\hline \multicolumn{12}{|l|}{ Total } \\
\hline Bajo Vinalopó & 22977 & 42134 & 44898 & 48405 & 54204 & 63324 & 74364 & 93810 & 148762 & 197261 & 222093 \\
\hline
\end{tabular}




\section{La ocupación antrópica del Valle y la lucha por los abastecimientos de agua}

Frente a esta marcada carencia de recursos hídricos la cuenca del Vinalopó ha presentado desde tiempos remotos una gran actividad antrópica y ello merced a que el eje hidrológico debe su origen a la configuración de una línea de fractura que con dirección NW-SE corta a las alineaciones béticas en el sector meridional de la provincia y que ha tenido un papel especial, en cuanto que ha constituido una excepcional vía de penetración y comunicación a lo largo de la historia desde las costas mediterráneas hacia el interior peninsular.

Ya desde el Paleolítico Medio y con el hombre de Neandertal se tienen noticias de poblamiento en la zona, que va a registrar una continua ocupación hasta nuestros días, en que nos encontramos que un 50\% de los municipios ha duplicado su población desde principios de siglo, y en algunos casos se ha llegado a triplicar o aumentaren diez veces el número de habitantes.

La penuria hídrica de la cuenca y la necesidad impuesta por los asentamientos han sido los desencadenantes de una dilatada historia de aprovechamientos de aguas regulados por costumbres e interferidos por pleitos y litigios. Los restos de obras hidráulicas, que se remontan a época romana, alcanzan sus mejores hitos en la época moderna, que al parecer recogió las ricas enseñanzas de los árabes. Ya en el siglo XIII se tienen noticias de intentos de trasvases, y obras representativas de la técnicas hidráulicas son los embalses de Elda (siglo XVII), Petrel (siglo XVIII) y Elche (siglo XVII). Será sin embargo el siglo XIX, con la expansión de las perforaciones, en principio, de mantos subálveos y, posteriormente, ya en el siglo XX, con la introducción de la energía eléctrica y el cribado de mantos más profundos, cuando se dispara el desequilibrio entre demandas y recursos.

Puede establecerse ya en el año 1940 los primeros signos de afecciones evidentes en las aguas subterráneas, con la bajada de caudales y desaparición de algunos manantiales, como la legendaria Fuente del Chopo en el Alto Vinalopó.

A comienzos de siglo la Unidad del Vinalopó constituía un enclave con una impronta agrarista muy acusada, pero en la que destacaba una dedicación fabril, cuyo origen artesanal venía supeditado a la elaboración de materias primas que producía el mismo suelo y a los trabajos relacionados con una agricultura de rendimientos escasos e inseguros.

La predominancia del secano, en el que la trilogía mediterránea era lo característico, unicamente se veía interrumpido por pequeñas huertas locales y campos regados, en los que los cultivos, a más de su aleatoriedad, tenían un marcado carácter autoconsumista. La inseguridad de esta agricultura determinó la dedicación hacia otras actividades complemetarias del agro, que eran realizadas bien por parte de los elementos no ocupados de las familias o en las épocas de paro estacional de la agricultura por todos los miembros. Esta condición, junto con el hecho de que el Vinalopó haya sido paso obligado en estas latitudes entre la meseta castellana y el mar y entre los llanos litorales y Valencia y Murcia, contribuyó desde un principio a crear un cierto hábito mercantil que apoyado en la agricultura árida e insegura determinará con el tiempo la proyección sectorial secundaria de esta cuenca, que en los últimos tiempos presenta altos índices de urbanización y terciarización.

En esta evolución participaron diversos factores, a cual más importante, de entre los que destacan el desarrollo demográfico y la evolución tecnológica sufrida en el último cuarto de siglo.

Este aspecto tuvo su máximo exponente en la aparición del ferrocarril y posteriormente en el proceso de electrificación de las ciudades, que ofrecía muestras incipientes del 
desarrollo de sus cascos urbanos. El sistema productivo había comenzado a modificarse y se necesitaban urgentemente nuevos recursos para el abastecimiento humano e industrial, $\mathrm{y}$, como no, agrario, para obtener productos alimenticios con que satisfacer las demandas de una población en crecimiento.

Los cambios geoeconómicos que se venían manifestando desde fines del siglo XIX, potenciados por las innovaciones técnicas del momento, contribuyeron a la introducción de trascendentales cambios en los sistemas de aprovechamiento de las aguas y por ende de la economía hídrica de la cuenca.

Los recursos superficiales autóctonos, insignificantes frente a las abultadas necesidades, empujan a una gran diversificación de los métodos y fuentes para conseguir nuevos caudales, que definen a la unidad como una de las más complejas en cuanto a los sistemas de aprovisionamiento y usos del agua.

El desarrollo de estos métodos de captación será desde fines del XIX una de las facetas más características de esta cuenca, que en las dos primeras décadas del siglo XX se ve acompañada en importancia por la llegada de aguas procedentes del desagüe del Segura mediante iniciativas acometidas a expensas de sociedades mercantiles privadas.

En materia de aguas subterráneas el Alto Vinalopó sigue siendo, igual que antaño lo era en aguas superficiales, la cuenca con recursos por excelencia, de manera que, como entonces, a expensas de sus recursos subsistirán un gran número de núcleos pertenecientes a otras subcuencas del Vinalopó y del enclave comarcal vecino del Campo de Alicante.

En el Bajo Vinalopó las iniciativas privadas buscan aguas en unidades hidrológicas ajenas. Frente a las ansiadas aguas del Júcar, buscadas desde el s. XIII, que no han llegado, se acomete la importación de aguas desde el Segura; que mucho más tarde, en la segunda mitad de siglo, será de nuevo el redentor de los secanos del Campo de Elche y del abastecimiento de los municipios de esta zona, merced a la llegada de las aguas del trasvase Tajo-Segura.

Estos logros no van a oscurecer en ningún caso las abundantes iniciativas de buscar caudales allí donde los hubiese para poder importarlos. El desarrollo inusitado de un buen número de asentamientos, más patente desde mediados de 1950, gracias a la urbanización e industrialización, y la reorientación agraria hacia el regadío en términos del Medio y Bajo Vinalopó, reclama nuevas soluciones a los déficits hídricos, que desde el primer momento cuentan con el agravante de un proceso de agotamiento por sobreexplotación y degradación de los escasos recursos locales, que a mayor abundancia se comparten fuera de la unidad.

Rebasada la década de los treinta, con la introducción de la energía eléctrica, los avances en las técnicas de prospección y el perfeccionamiento en la fabricación de bombas de turbinas o rodetes, se permite la extracción de aguas a mucha más profundidad, lo que conlleva desde mediados de la centuria un activo cribado de los mantos acuíferos, que a fines de los setenta presentaban ya claras afecciones.

Las aguas profundas fueron elevadas en principio por iniciativas privadas en mayor medida, que desde ese momento vieron una gran ventaja en este sistema de obtención de aguas, no sujeto a una normativa legal, ya que esas aguas eran de propiedad particular,y además de abastecer sus necesidades podían comerciar con ellas, obteniendo de este modo altos rendimientos.

La venta del agua y su distribución variaba en función de que se tratara de empresas particulares o de comunidades de riego. En ambos casos, y ya desde principios de siglo, se había establecido una amplia infraestructura y normativa de riego que se fue desarrollando posteriormente, y en la que de ordinario se participaba en la compra o disfrute del agua mediante «acciones y horas de agua», que se insertaban en ocasiones en un antiguo sistema 


\begin{tabular}{|lrrrr|}
\hline Municipio: & $\begin{array}{c}\text { Superficie } \\
\text { en } \mathbf{K m}^{2}\end{array}$ & $\begin{array}{c}\text { \% del } \\
\text { total }\end{array}$ & Habitantes & $\begin{array}{r}\text { Densidad } \\
\text { demográfica }\end{array}$ \\
\hline Bañeres & 49,5 & 2,50 & 7.085 & 143,13 \\
Benejama & 34,6 & 1,74 & 1.850 & 53,46 \\
Biar & 97,9 & 4,95 & 3.412 & 34,85 \\
Campo de Mirra & 21,3 & 1,07 & 396 & 18,59 \\
Cañada & 19,9 & 1,00 & 1.058 & 53,16 \\
Sax & 68,0 & 3,43 & 8.309 & 122,19 \\
Villena & 344,2 & 17,38 & 31.037 & 90,17 \\
Aspe & 69,8 & 3,52 & 16.008 & 229,34 \\
Elda & 44,7 & 2,25 & 57.515 & $1.286,68$ \\
Hondón de los & & & & \\
Frailes & 12,5 & 0,63 & 554 & 44,32 \\
Hondón de las & & & & \\
Nieves & 68,9 & 3,48 & 1.517 & 22,01 \\
La Algueña & 18,4 & 0,92 & 1.527 & 82,98 \\
La Romana & 43,6 & 2,20 & 1.971 & 45,20 \\
Monforte & 79,2 & 4,00 & 4.943 & 62,41 \\
Monovar & 151,8 & 7,66 & 12.122 & 79,85 \\
Novelda & 76,0 & 3,83 & 22.288 & 293,26 \\
Petrel & 104,3 & 5,27 & 23.591 & 226,18 \\
Pinoso & 126,0 & 6,36 & 5.674 & 45,03 \\
Salinas & 61,9 & 3,12 & 1.096 & 17,70 \\
Crevillente & 103,3 & 5,22 & 22.456 & 217,38 \\
Elche & 325,8 & 16,45 & 184.912 & 567,56 \\
Santa Pola & 57,9 & 2,92 & 14.725 & 254,31 \\
& & & & \\
\hline
\end{tabular}

de subastas.

Desde inicios de la segunda mitad de siglo se introducen regulaciones en relación a los aprovechamientos de aguas subterráneas. La legislación de colonización promulgada tras la Guerra Civil, y en particular el Decreto de 5 de febrero de 1954, declaraba de alto interés nacional los trabajos, obras e instalaciones que para la investigación y alumbramiento de las aguas subtarrénas con fines exclusivos de riego realizase el Instituto Nacional de Colonización, en cumplimiento de la base 19 de la Ley de 26 de diciembre de 1939. Mediante esta nueva regulación, las obras de captación y alumbramiento realizadas por el Instituto se consideraban de utilidad pública, organismo que las podía ceder a particulares y entidades privadas, que pasaban a beneficiarse de ellas, como de hecho ocurrió con un buen número de pozos.

En este marco propicio coinciden otros factores concretos que permiten que el regadío se incremente en general en toda la cuenca y alcance cotas inverosímiles, como lo son incrementos del 303\% en el Medio Vinalopó.

Los municipios y sus núcleos urbanos alcanzan, asimismo, cotas de expansión en algunos casos inusitadas y empieza a perfilarse el desarrollo de los núcleos litorales de Elche y Santa Pola, en los que cada vez es más evidente el desarrollo del uso residencial y 
turístico, que incrementa las demandas.

La regulación de los abastecimientos urbanos mediante la Ley de Bases de Régimen Local, de 24 de junio de 1955, introducía las normas para los auxilios del Estado a los Ayuntamientos en las obras de abastecimiento de agua potable, que motivó, entre otras cuestiones, la llegada de las aguas del Taibilla (de la vecina cuenca del Segura) a los municipios del Bajo Vinalopó, y la perforación de pozos para dotar de agua a los municipios, realizadas por el S.G.O.P. y el I.G.M.E.

Será este último aspecto el más problemático, puesto que a pesar de las directrices públicas, la falta de capacidad jurídica del Estado ante la separación antinatural de las aguas superficiales y subterráneas determinó que la iniciativa privada fuera la que en mayor medida cubriera la demanda de los abastecimientos, tanto al agro como a los núcleos urbanos, provocando una desarrollo inusitado de las perforaciones a cargo de particulares y empresas asociadas, que han llevado, ante la inexistencia de otros recursos, a un gran número de afecciones tras un período desarrollista en el que la falta de planificaciones ha comportado cotas irracionales de consumo, degradación y agotamiento.

\section{Acuíferos sobreexplotados}

Resultado evidente de los hechos antes expuestos es la declaración de sobreexplotados de dos acuíferos de la cuenca, el de Jumilla-Villena y el de la Sierra de Crevillente.

El acuífero de Jumilla-Villena se extiende desde el núcleo urbano de Jumilla, al oeste, hasta la depresión triásica de Villena, al este; el límite norte viene establecido por las sierras del Buey, del Príncipe y Lácera, y el límite sur por las del Carche, Pansas, Serral, Teja y estribaciones septentrionales de las sierras de Salinas y el Collado.

Las rocas permeables ocupan $340 \mathrm{~km}^{2}$, y las principales son las dolomías y calizas del Cretácico Superior, con un espesor máximo de 550 metros. En continuidad hidráulica sobre las anteriores aparecen, en ocasiones, calcarenitas del Mioceno y gravas y arenas del Cuaternario.

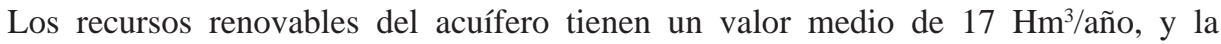
descarga, que se lleva a cabo de forma exclusiva, mediante bombeos en unos cincuenta pozos, asciende a $34 \mathrm{Hm}^{3} /$ año. Ante tal desajuste es comprensible que se den descensos del nivel piezométrico, que se cifran en 2,5 por año, lo que está en relación con una sobreexplotación media anual del acuífero entre 8 y $18 \mathrm{Hm}^{3}$ por año, correspondientes a una evolución comprobada desde 1975 (vide gráfico 3).

La mayor garantía, a pesar de lo dicho, estriba en que posee unas reservas elevadas, de unos $3.200 \mathrm{Hm}^{3}$. Además, el acuífero tiene, en general, aguas de buena calidad, salvo en las proximidades de los límites este y oeste, debido a la cercanía de materiales triásicos salinos.

El agua extraída se utiliza en un $72 \%\left(24,2 \mathrm{Hm}^{3} /\right.$ año $)$ a cubrir la demanda agrícola, y el $28 \%\left(9,5 \mathrm{Hm}^{3} /\right.$ año $)$ restante al abastecimiento urbano e industrial. Pero quizás el dato más significativo es que un $80 \%$ se utiliza fuera de sus límites, contribuyéndo a satisfacer parte de la demanda agrícola de los términos municipales de Jumilla, Villena, Elda, Sax, Petrel, Monforte del Cid, Elche, Agost, San Vicente del Raspeig, Muchamiel, San Juan, Campello y Alicante. Así mismo atiende total o parcialmente el abastecimiento de las poblaciones de Raspay y Jumilla (Murcia), y las de Elda, Petrel, Novelda, Agost, Muchamiel y Campello (Alicante).

En su interior, se aplica en parte a los regadíos de los términos municipales de Yecla, Villena y Jumilla, cubriéndo en un $40 \%$ el abastecimiento urbano de Jumilla. 
GRÁFICO 3. Isodescensos del nivel piezométrico del acuífero Jumilla-Villena. FuENTE: Las aguas subterráneas en la provincia de Alicante. I.T.G.M.E. 1982.

En grupo aparte se sitúa el complejo Subbético de la Sierra de Crevillente, que con una superficie restringida, apenas $200 \mathrm{~km}^{2}$, queda arrinconado en el sector occidental. En él la litología es dolomítico-caliza, pero la escasa alimentación y la reducida extensión del sistema, así como su complejidad estructural, comprometen el balance del mismo, sometido a grandes extracciones que hacen descender continuamente sus niveles.

El acuífero se sitúa entre los límites orográficos de la Sierra de Crevillente, al sur, las de Argallat, Cava y Rollo, al norte, el límite provincial entre Alicante y Murcia, al oeste, y las faldas orientales de las sierras de Ofra y Ors al este.

Las dolomías y calizas masivas del Lias Inferior, con una potencia conjunta de 350 metros, constituyen la principal roca permeable. El impermeable de base lo forman arcillas y yesos del Trias. El acuífero tiene una extensión de $90 \mathrm{~km}^{2}$.

Los recursos renovables del mismo se han calculado en $2 \mathrm{Hm}^{3}$, procedentes de la infiltración del agua de lluvia; mientras que su descarga, que se produce exclusivamente por bombeos en 19 pozos, se sitúa, en los últimos años, en $16 \mathrm{Hm}^{3}$. Se mantiene por ello una importante sobreexplotación, que ha oscilado entre 13 y $19 \mathrm{Hm}^{3} /$ año, lo que ha provocado descensos en los niveles del orden de 6 metros en el sector occidental y 19 en el oriental (vide gráfico 4).

Las reservas explotables del acuífero en el límite rentable de la extracción se han calculado en $147 \mathrm{Hm}^{3}$, por lo que se encuentra en grave peligro la explotación, si tenemos, además en cuenta que el total de sólidos disueltos oscila entre 1,6 y 1,9 gm/l en las áreas de mayor concentración de pozos (galería de Los Suizos-El Tolomó), mientras que en otros sectores se sitúa en torno a 1,3 y $0,6 \mathrm{mg} / \mathrm{l}$.

El agua extraída se destina en un $84 \%$ a cubrir la demanda agrícola $\left(12,7 \mathrm{~m}^{3}\right)$ y en un 
GRáfico 4. Curva de explotación del acuífero de la Sierra de Crevillente. FuENTE: M.O.P.U. 1989.

$16 \%\left(2,35 \mathrm{~m}^{3}\right)$ para el abastecimiento industrial y urbano. De esos volúmenes el $78 \%$ se utiliza fuera de sus límites, con áreas de regadío en Aspe, Hondón de las Nieves, Elche, Crevillente, Albatera, Orihuela y Hondón de los Frailes. Los núcleos abastecidos son los de Aspe, Hondón de las Nieves y Hondón de los Frailes. Asimismo, cubre las demandas de la población diseminada, y para industria y riego de pequeñas parcelas en las zonas de Elche y Crevillente.

A la vista de los balances hídricos expuestos para sendas formaciones acuíferas, resulta evidente que existe un alto nivel de «sobreexplotación». Este concepto, introducido hace sólo dos décadas en nuestro país y aplicado en principio a sectores muy concretos (Baleares), no es entendido de igual forma por todos, y llega incluso a ser entre ciertos autores un hecho necesario para sostener el desarrollo.

El Reglamento del Dominio Público Hidráulico aprobado por el Real Decreto 849/ 1986, de 11 de abril, establece en el punto 2 de artículo 1, que:

«Se considerará que un acuífero está sobreexplotado o en riesgo de estarlo cuando se está poniendo en peligro inmediato la subsistencia de los aprovechamientos existentes en el mismo, como consecuencia de venirse realizando extracciones anuales superiores o muy próximas al volúmen medio de los recursos anuales renovables, o que produzcan un deterioro grave de la calidad de agua.

La existencia de riesgo de sobreexplotación se apreciará también cuando la cuantía de las extracciones, referida a los recursos renovables del acuífero, genere una evolución de éste que ponga en peligro la subsistencia a largo plazo de sus aprovechamientos». 
La definición de acuíferos sobreexplotados que efectua el RDPH en su artículo 171.2 implica situaciones inminentes o a largo plazo, lo que implicaría en el segundo caso que cualquier acuífero, dentro de las nuevas directrices del Plan Hidrológico Nacional, debería contar con normas de explotación adecuadas, a tenor de su estado actual y de los procesos de desarrollo ulteriores. Con este concepto la Administración Pública del agua hace previsión de un problema y pretende anticiparse a él, pudiendo establecerse la «declaración provisional de sobreexplotación».

La Dirección General de Obras Hidráulicas resolvió con fecha 31 de julio de 1987, declarar provisionalmente sobreexplotados los acuíferos Sierra de Crevillente y JumillaVillena. Declaraciones que se realizaron a tenor de la propuesta elevada al respecto por las Confederaciones Hidrográficas del Júcar y del Segura, que elaboraron conjuntamente sendos estudios sobre la situación de ambos acuíferos, en los que, con base a los informes efectuados por el Instituto Geológico y Minero de España, quedaba plenamente justificada dicha declaración.

Es sintomático el hecho de que la declaración de sobreexplotación iniciada por las Juntas de Gobierno de las Confederaciones Hidrográficas lo fuera de oficio por parte de la Administración, y no a instancias de los usuarios, que venían sufriendo las consecuencias de la sobreexplotación hacía ya algún tiempo. En este hecho podemos entrever una profunda problemática que no ha conseguido eliminar aún la nueva Ley de Aguas, la figura de los «dueños o propietarios del agua», que habitualmente no son los que las utilizan, ya que los usuarios son otros, entre los que se citan regantes y consumidores urbanos, residenciales e industriales. Es, por ello obvio que en la declaración de sobreexplotación no convergen los puntos de vista ni los intereses de estas dos figuras, bien diferentes.

Una vez efectuada dicha «declaración provisional de sobreexplotación», y de acuerdo al artículo171.4, quedaría señalado el perímetro de la zona afectada, expresado ya en el estudio preeliminar, y llevaría aparejados, dentro de su ámbito, los siguientes efectos:

a) Paralización de todos los expedientes de autorización, de investigación o de concesión de aguas subterráneas.

b) Suspensión del derecho de apertura de nuevas captaciones establecido en el artículo 52.2 de la Ley de Aguas (aprovechamientos de volúmen de agua subterránea que no sobrepasen los $7.000 \mathrm{~m}^{3} / \mathrm{a} \tilde{n}$ ), y que requerirá de autorización expresa.

c) Detención de todos los expedientes en trámite de modificación de características de las concesiones de aguas subterráneas.

d) Constitución forzosa de la Comunidad de Usuarios del acuífero, de no existir, por aplicación del artículo 79 de la citada norma.

Llevados a cabo dichos preliminares y de acuerdo con el art. 171.5 del Reglamento: «... el organismo de cuenca, oída la Comunidad de Usuarios del acuífeo, elaborará un plan de ordenación de las extracciones para lograr su explotación más racional, y, en orden a conseguir la superación de los problemas planteados ...».

Este plan será sometido a información pública y a dictamen del Consejo del Agua de la cuenca. En los casos que ahora analizamos la aprobación corresponde a la Dirección General de Obras Hidráulicas, por tratarse de acuíferos compartidos entre las Confederaciones Hidrográficas del Júcar y del Segura.

Según reza en el artículo 171.6 del R.D.P.H., una vez aprobado el Plan de Ordenación se supondrá la declaración definitiva de sobreexplotación de acuífero. Declaración que implicará la ejecución inmediata del plan.

\section{Los planes de ordenación}


La declaración de sobreexplotación y la ordenación de extracciones se conciben como instrumentos suficientes y eficaces para elaborar un plan y resolver así un conflicto que pudiera plantearse. Sin embargo, la situación actual, en la que se encuentran ciertas cuencas, no es justamente la idílica para hablar de anticipación a un problema sino, más bien, la de actuaciones inminentes, en las que la ordenación de las extracciones, dada la gravedad en que se encuentran estos acuíferos supondría la práctica reducción de una buena parte de ellos.

Es por ello que los planes de ordenación se esten elaborando por propia necesidad en condiciones muy distintas a las imaginadas en la concepción de la ley. Es decir, sobre acuíferos realmente sobreexplotados, definidos como «acuíferos en situación terminal», en los que no cabe buscar soluciones óptimas. Es más, dada la delicada situación de estos acuíferos, en los que se está funcionando desde hace tiempo sobre las reservas, en auténtica extracción minera del agua, y en la que la privación o reducción de estos recursos conllevaría daños sociales importantes, se hacen necesarias otras actuaciones que, si bien al margen del concepto genuino de un plan de ordenación, son de otra parte inherentes a la propia legislación hidráulica y a los objetivos generales de toda la planificación hidrológica, de la que la ordenación de acuíferos es un caso puntual.

Este primer principio de planificación hidrológica entraña nuevos conceptos de interés general frente a explotaciones privativas e individualizadas. Sin embargo, la pervivencia de títulos jurídicos diferentes, representados en las figuras de los «propietarios de agua», frente a los «usuarios» de las mismas, que tienen además intereses muy contrastados, impide en gran medida la consecución de los planes de ordenación, exclavos en mayor medida de cuestiones legales y jurídicas más que técnicas o económicas.

La elaboración de estos planes de ordenación y su ejecución tropieza de este modo con la propia pasividad, e incluso presunta ignorancia, de los usuarios especialmente afectados por la sobreexplotación, ya que la petición o denuncia no llega a la Administración, que acaba interviniendo de oficio.

Los Planes de Ordenación conllevan unas actuaciones básicas o generales que han quedado reflejadas en las propuestas para los casos que nos ocupan, Crevillente y JumillaVillena;

1. Aprobación de los nuevos perímetros, identificados por vértices, que delimitan las zonas afectadas, dentro de los cuales será de aplicación la normativa que desarrolla el artículo 171 del RDPH.

2. Establecimiento de una red de control piezométrico y otras de calidad del agua, que permitan conocer puntual y fielmente la evolución en el tiempo y en el espacio de los aspectos cuantitativos y cualitativos de las aguas del acuífero.

3. Los volúmenes de agua a conceder a los usuarios del acuífero, hasta la puesta en marcha de alternativas que liberen en todo o en parte los caudales asignados, tendrán como límite la explotación tipo considerada. Esta quedará reducida al valor de la aportación externa en cuanto le lleguen caudales ajenos al sector y se proceda a la clausura de aprovechamientos que se crea necesaria. Al objeto de controlar efectivamente la cuantía de estos volúmenes se instalarán aparatos de medida a la salida de todas las captaciones.

4. Fuera de las investigaciones que promueva la Administración Hidráulica existe la prohibición absoluta de efectuar dentro del perímetro de delimitación del acuífero cualquier operación destinada a determinar la existencia de aguas subterráneas.

5. Reordenación de las extracciones del acuífero, que consiste en sustituir pozos de las áreas sometidas a fuertes limitaciones de caudal, profundidad del agua y contamina- 
ción salina por otros ubicados en zonas más aptas de los acuíferos. Sustituciones que deben ser aprobadas por la Junta de Explotación del acuífero.

6. El amojonamiento de la zona de regadío es una actuación que en concreto se ha efectuado en las áreas de Aspe, Hondón de las Nieves, Elche, Crevillente, Orihuela y Hondón de los Frailes, y en relación con el acuífero de Crevillente. Medida que cercena la expansión de áreas consideradas como regables, que no estan cultivadas e incluidas en anteriores perímetros, y que presuponen nominalmente mayores demandas.

Existen otras medidas, adoptadas dentro de un plan de mínimos, que son complementarias a la ordenación de las extracciones y cuyo fin es la liberación parcial de caudales del acuífero mediante la incorporación de recursos ajenos al mismo, que se concretan en las aguas residuales interiores y próximas a los mismos y los recursos que aporten sistemas de abastecimiento principales, estos últimos en aplicación de lo establecido en el artículo 26.1 de la Ley de Bases de Régimen Local;

1. Por lo que concierne a las aguas residuales en el acuífero de Crevillente, las áreas comprendidas en Aspe, Hondón de las Nieves y Hondón de los Frailes se beneficiarían de recursos generados por estos mismos núcleos urbanos, así como por las procedentes y sobrantes de la depuradora de Alicante (Rincón de León).

2. En el caso del acuífero de Jumilla-Villena, reutilizaría las de Elda para la SAT número 968 de Elda, que actualmente se surte de dicho acuífero, y las residuales de Villena, que irían tanto a la Comunidad de Regantes de la Huerta como, de existir sobrantes, a las Comunidades que las soliciten. En ambos casos se requiere un sistema de infraestructuras de canales y de embalses para conseguir mayores diluciones de las aguas que se mezclan con las procedentes de los pozos.

3. Liberar al Sistema de Crevillente del abastecimiento que efectua a la zona industrial y urbana de Elche-Crevillente y obtener las autorizaciones definitivas de abastecimiento por parte de la Mancomunidad de Canales del Taibilla a los municipios de Aspe y Hondón de las Nieves, son otras de las actuaciones que requieren el desarrollo de la Ley de Bases citada y la modificación de la vigente Ley, de 27.4.1946, de la M.C.T.

Estas actuaciones complementarias no parecen estar necesariamente vinculadas a los Planes de Ordenación de los acuíferos, en los que no existe una indicación clara respecto a su contenido, salvo en su declaración genérica de intenciones, en la que se dice que el plan contemplará la «ordenación de todas las extracciones para lograr su explotación más racional».

Por último, entre medidas más amplias, que tienen efectos a mayor escala territorial y a más largo plazo, se citan además de los últimos hechos apuntados, la inclusión de toda la zona en una problemática más amplia, que afecta a toda la cuenca del Vinalopó, como es la solución de aportes foráneos en los esquemas de trasvases del proyecto «Canal del Júcar-Marinas-Vinalopó»; o la propia redelimitación de la zona regable de Riegos de Levante M.I., en la que se incorporarían áreas de Elche, Crevillente, Albatera y Orihuela que hoy se abastecen del Sistema de Crevillente y que pasarían a ser atendidas por caudales procedentes del trasvase Tajo-Segura, así como de los propios sobrantes del Segura y azarbes.

De mediar estrictos Planes de Ordenación de extracciones los aprovechamientos no podrían mantenerse de forma indefinida, lo que conllevaría la reducción y práctica desaparición en muchos casos del aprovechamiento. Reducción que de forma natural se va a producir de no intervenir en la gestión de su explotación, de tal suerte que estos Planes son auténticos planes de «priorización de aprovechamientos»y «maximización de expectati- 
vas». Incluso se puede hablar de la introducción de un cierto orden en un proceso terminal y traumático que en todo caso se producirá con independencia de que intervenga o no la Administración.

Volviendo a los planes de mínimos o actuaciones básicas antes expuestos, no son en definitiva más que el prolegómeno, durante la fase transitoria en que nos situamos y por lo tanto el camino preliminar, para abordar soluciones de carácter definitivo ya en el marco del Plan Hidrológico.

Con todo, los impedimentos que comportan aspectos jurídicos y derechos consuetudinarios deberán ser salvados y resueltos, pues en estos momentos continuan siendo la traba esencial, que comienza por impedir la configuración de un elemento esencial como es la Comunidad de Usuarios, requisito básico para desarrollar un Plan de Ordenación, y que en la actualidad no se ha constituido aún en los dos acuíferos sobreexplotados.

\section{Bibliografía}

ARAGONÉS BELTRÁN, J. M. et alteri: «Planes de Ordenación de acuíferos sobreexplotados: la experiencia de la Sierra de Crevillente (Alicante)». La sobreexplotación de acuíferos. Almería, 1989, pp. 177-191.

BRU RONDA, C.: «El trasvase Júcar-Vinalopó». Demanda y economía del agua en España. Alicante. CAM, 1988. 13 pp.

- «Las aguas subterráneas en la provincia de Alicante». Geografía de la provincia de Alicante. Tomo I.1. Ediciones Mediterráneo. 1988.

- Los caminos del agua: el Vinalopó. Confederación Hidrográfica del Júcar. Valencia. 1992.

- Recursos, usos y economía del agua en la provincia de Alicante. Tesis Doctoral. Instituto de Geografía de la Universidad de Alicante. 1986. (En prensa).

CABEZAS CALVO-RUBIO, F. et alteri: «Metodología de los estudios destinados a la elaboración de los planes de ordenación de los acuíferos Alto y Bajo Guadalentín, Cresta del Gallo y AscoySopalmo (cuenca del Segura. España)». XXIII Congreso A.I.H. Sobreexplotación de acuíferos. Puerto de la Cruz, 1991, pp. 351-354.

CORCHON RODRÍGUEZ, F. et alteri: «Datos básicos para la realización del plan de ordenación del acuífero sobreexplotado Sierra de Crevillente». La sobreexplotación de acuíferos. Almería. 1989, pp. 471-483.

I.G.M.E.-DIPUTACIÓN PROVINCIAL DE ALICANTE: Las aguas subterráneas en la provincia de Alicante. 1982. Mecanograf.

I.T.G.M.E.: Las aguas subterráneas en la Comunidad Valenciana. Valencia. 1986. 298 pp.

M.O.P.T.: Plan Hidrológico. Proyecto de Directrices. Confederación Hidrográfica del Júcar. Junio de 1992.

M.O.P.U.: Estudio de actualización de la explotación, inventario de puntos de agua y propuesta de ordenación de las extracciones del acuífero Jumilla-Villena. Dirección General de Obras Hidráulicas. Valencia. 1989.

M.O.P.U.: Estudio de la explotación, inventario de puntos de agua y propuesta de ordenación de las extracciones del acuífero Sierra de Crevillente. Dirección General de Obras Hidráulicas. Valencia. $1989.31 \mathrm{pp}$.

PULIDO BOSCH, A.: «The overexploitation of sowe karstic. Aquifers in the province of Alicante (Spain)». XXIII Congreso A.I.H. Sobreexplotación de acuíferos. Puerto de la Cruz. 1991, pp. 557-561.

REVERTE NAVARRO, A. y PÉREZ PÉREZ, E. (Editores): Legislación de Aguas. Tecnos. Madrid, 1990. Cuarta edición. 